\title{
Assessing leanness level with demand dynamics in a multi-stage production system
}

\author{
Rehab Ali \\ Department of Management of Technology, Nile University, Giza, Egypt, and \\ Ahmed Deif \\ Department of Industrial Technology and Packaging, \\ California Polytechnic State University, San Luis Obispo, California, USA
}

\begin{abstract}
Purpose - The purpose of this paper is to present a dynamic model to measure the degree of system's leanness under dynamic demand conditions using a novel integrated metric.

Design/methodology/approach - The multi-stage production system model is based on a system dynamics approach. The leanness level is measured using a new developed integrated metric that combines efficiency, WIP performance as well as service level. The analysis includes design of experiment technique at the initial analysis to examine the most significant parameters impacting the leanness score and then followed by examining different dynamic demand scenarios. Two scenarios were examined: one focussed low demand variation with various means (testing the impact of demand volumes) while the second focussed on high demand variation with constant means (testing the impact of demand variability). Findings - Results using the data from a real case study indicated that given the model parameters, demand rate has the highest impact on leanness score dynamics. The next phase of the analysis thus focussed on investigating the effect of demand dynamics on the leanness score. The analysis highlighted the different effects of demand variability and volumes on the leanness score and its different components leading to various demand and production management recommendations in this dynamic environment. Research limitations/implications - The presented lean management policies and recommendations are verified within the scope of similar systems to the considered company in terms of manufacturing settings and demand environment. Further research will be carried to extend the dynamic model to other dynamic manufacturing and service settings.

Practical implications - The developed metric can be used not only to assess the leanness level of the systems which is very critical to lean practitioners but also can be used to track lean implementation progress. In addition, the presented analysis outlined various demand management as well as lean implementation policies that can improve the system leanness level and overall performance.

Originality/value - The presented research develops a novel integrated metric and adds to the few literature on dynamic analysis of lean systems. Furthermore, the conducted analysis revealed some new aspects in understanding the relation between demand (variability and volume) and the leanness level of the systems. This will aid lean practitioners to set better demand and production management policies in today's dynamic environment as well as take better decisions concerning lean technology investments.
\end{abstract} Keywords Lean manufacturing, Manufacturing management, Demand management Paper type Research paper

\section{Nomenclature}

$\operatorname{AOUT}(t)$

actual output at time $t$

AQL

$\mathrm{B}(t)$

$\mathrm{B}_{0}(t)$

$\operatorname{COT}(t)$ acceptable quality level

backlog level at time $t$

initial backlog at time $t$

changeover time at time $t$
$\mathrm{CT}_{i}(t)$

$\mathrm{D}(t)$

$\mathrm{DD}(t)$

$\mathrm{DR}(t)$

$\operatorname{DSR}(t)$ cycle time at time $t$ for station $i$ number of defects at time $t$ delivery delay at time $t$ demand rate at time $t$ desired shipment rate at time $t$ 


\begin{tabular}{|c|c|c|c|}
\hline DT & delay time (due to quality) & $\mathrm{PR}_{i}(t)$ & production rate at time $t$ for \\
\hline $\mathrm{FGI}(t)$ & finished goods inventory at time $t$ & & station $i$ \\
\hline $\operatorname{FOR}(t)$ & filled order rate at time $t$ & $\mathrm{QC}(t)$ & quality control level at time $t$ \\
\hline $\mathrm{FR}(t)$ & finishing rate at time $t$ & $\mathrm{QCOR}(t)$ & quality control output rate at \\
\hline $\mathrm{IT}(t)$ & inspection time at time $t$ & & time $t$ \\
\hline $\mathrm{LS}(t)$ & leanness score at time $t$ & $\mathrm{QCSR}(t)$ & quality control start rate at time $t$ \\
\hline $\mathrm{MA}(t)$ & machine availability at time $t$ & $\mathrm{QR}(t)$ & quality rate at time $t$ \\
\hline $\operatorname{MOPT}(t)$ & $\begin{array}{l}\text { minimum order processing time } \\
\text { at time } t\end{array}$ & $\begin{array}{l}\mathrm{QS}(t) \\
\mathrm{SPT}\end{array}$ & $\begin{array}{l}\text { quality signal at time } t \\
\text { scheduled production time }\end{array}$ \\
\hline $\operatorname{MSR}(t)$ & maximum shipment rate at time $t$ & $\mathrm{SR}(t)$ & shipment rate at time $t$ \\
\hline NAT & net available time & TDD & target delivery delay \\
\hline $\operatorname{NOT}(t)$ & net operating time at time $t$ & TGWIP $(t)$ & target WIP at time $t$ \\
\hline $\mathrm{OEE}(t)$ & $\begin{array}{l}\text { overall equipment effectiveness } \\
\text { at time } t\end{array}$ & $\begin{array}{l}\text { THCT } \\
\text { THOUT }(t)\end{array}$ & $\begin{array}{l}\text { theoretical cycle time } \\
\text { theoretical output at time } t\end{array}$ \\
\hline $\operatorname{OSL}(t)$ & overall service level at time $t$ & $\operatorname{TWIP}(t)$ & total WIP at time $t$ \\
\hline $\mathrm{OWE}(t)$ & overall WIP efficiency at time $t$ & $\operatorname{UPDT}(t)$ & unplanned downtime at time $t$ \\
\hline PDT & planned downtime & $\mathrm{WIP}_{i}(t)$ & WIP level at time $t$ for station $i$ \\
\hline $\mathrm{PE}(t)$ & performance efficiency at time $t$ & & \\
\hline $\operatorname{PIR}(t)$ & roduction input rate at time $t$ & & \\
\hline
\end{tabular}

\section{Introduction}

Due to global competition and market dynamics, companies all over the world are under tremendous pressure to reduce their costs and increase their service level. There are many tools and strategies that manufacturing organizations can implement to achieve these goals. Lean manufacturing is one of the strategies that was implemented by many companies in their effort to attain the shortest cycle time and reduce costs by eliminating different wastes in their systems. The success of companies that adopted lean principles has increased the interest toward lean manufacturing. The companies and organizations that are in other areas such as the service industry and the healthcare sector are also applying lean principles to reduce their cost and improve their systems. Although many companies are interested in lean and trying to implement lean tools, the percentage of companies that have a successful lean initiative is not very high. According to Christopher (2000), lean works best in high volume, low variety and predictable environments. However, reality reveals that today's manufacturing settings are faced with different dynamics and uncertainties than these ideal conditions. An example of such uncertainty is the customer demand. Demand dynamics is a critical parameter in today's environment to sustain customer satisfaction and keep competitive edge for firms. Minor fluctuations in demand at the end-user or the retail level cause high variation in demand for upstream point in the supply chain. This is referred to as the Bullwhip effect.

This paper addresses two important aspects in lean systems' implementation. The first aspect is how to measure the leanness level from an integrated and dynamic perspective. Second, the paper aims at studying the impact of demand uncertainty on the leanness level dynamics. The approach starts with proposing a new dynamic model to measure the degree of leanness using system dynamics (SD) approach. This is followed by a design of experiments (DOE) technique to test the different model parameters significance on the developed integrated leanness score. Finally, the impact of different demand uncertainty 
scenarios are investigated to provide insights and recommendations for managing both internal lean parameters as well as external demand management decisions to secure a successful lean implementation.

\section{Literature review}

Research about lean systems, philosophies, tools and techniques can be found in Womack and Jones (2003), Black (2007), Abdulmalek and Rajgopal (2007), Staats et al. (2011), Elsayed et al. (2013) and Yang et al. (2015). The review in this section will focus on leanness assessment as well as dynamic analysis of manufacturing systems using SD since both topics are related to the proposed work.

Examples of research work-related leanness level assessment include a checklist of 36 indicators that was developed to assess the changes toward lean manufacturing by Sanchez and Perez (2001). Using the results from a survey for manufacturing plants located in the Spanish region of Aragon, they analyzed which lean production indicators were more used to assess the company's improvements in their production systems, and the determinants on the use of these indicators. They divided these indicators into six groups which reflect the major characteristics of lean as: elimination of zero value activities, multifunctional teams, continuous improvement, production and delivery JIT, supplier integration and flexible information system. Savsar and Al-Jawini (1995) used simulation to analyze the performance of just-in-time production system. The model measured the effects of random processing times, numbers of kanbans between stations, variability in demand, line length and kanban operating policies on some system performance measures, such as throughput rate, work-inprocess inventory and station utilizations. Elnadi and Shehab (2014) presented a conceptual model that can be used in measuring the degree of product-service system (PSS) leanness in UK manufacturing companies. The model assessed PSS leanness based on five lean enablers (supplier relationship, management leanness, workforce leanness, process excellence and customer relationship), 21 criteria (supplier delivery, culture of management, process optimization, etc.) and finally 73 attributes. Detty and Yingling (2000) used discrete event simulation as a tool to support organizations with the decision to implement lean manufacturing through quantifying the benefits achieved from applying lean principles. The current status of lean production assessment in China was also evaluated by Taj (2008). Nine key areas of manufacturing were evaluated in this assessment which were: inventory; team approach; processes; maintenance; layout/handling; suppliers; setups; quality; and scheduling and control. The results were displayed in the score worksheet and a lean profile chart was created to display the status of the plant and the gaps between the current situation and their specific lean targets. Almomani et al. (2014) proposed an integrated model of lean assessment and analytical hierarchy process (AHP) to define the route of lean implementation based on the perspective priorities for improvement. AHP was used to set the priorities of implementing lean in the different perspectives of the enterprise, according to the following criteria: lean radar score, cost of implementation, financial gains, time of completion, technological and administrative obstacles, and the degree of risk involved. Quality, cost, delivery, and motivation key performance indicators were used to measure the improvements achieved via applying this approach. Wan et al. (2007) used data envelopment analysis techniques to pinpoint the leanness frontier as a benchmark for the leanness score. The resulting leanness score indicated "how lean the system is" or "how much leaner it can become." The metrics used in the analysis were time and cost based. In addition, Pakdil and Leonard (2014) developed a comprehensive 
tool called the leanness assessment tool (LAT) using both quantitative (directly measurable and objective) and qualitative (perceptions of individuals) approaches to assess lean implementation. The LAT measured leanness using eight quantitative performance metrics: time effectiveness, quality, process, cost, human resources, delivery, customer and inventory. The previous metrics were captured using fuzzy membership function highlighting both improvement successes and needs in lean implementation. Fuzzy logic was further used in many researches as a measuring tool for lean production. Behrouzi and Wong (2011) developed fuzzy membership functions to assess the lean performance in manufacturing systems. It supported an efficient measurement of lean performance by producing a final integrated unit-less score. Susilawati et al. (2015) proposed a procedure for measuring degree of lean application which combines the advantage of the various procedures and improve the weakness of various procedures. The procedure used fuzzy number for scoring degree of application of lean practices focussing in qualitative factors like culture and satisfaction.

Various approaches were used to dynamically model and analyze manufacturing systems including SD introduced by Forrester (1961). Examples of dynamic analysis of manufacturing systems using SD is the three-echelon production distribution system used as a supply chain reference model for comparing various methods of improving total dynamic performance by Wikner et al. (1991). Helo (2000) shows how agility is built into supply chains. Three simulation models were studied: the demand magnification effect in supply chain, the analysis of capacity surge effects, and the trade-off between capacity utilization and lead times. The analysis recommended smaller order sizes, echelon synchronization and capacity analysis as methods of improving the responsiveness of a supply chain. Poles (2013) explored the interaction between the physical flow, information flows and company policies to generate the dynamics of the re-manufacturing process. Deif and ElMaraghy (2014) presented dynamic systems approach to study challenges of implementing production leveling and its associated costs in a lean cell producing at takt time. Results showed that determining the most feasible leveling policy is highly dictated by both the cost and limitations of capacity scalability. In addition, delivery sequence plans of different products/parts is needed to achieve mix leveling and lot sizes affect the feasible production leveling policy while implementing lean principles. Zhang et al. (2012) used system engineering concept to compare the SD models of traditional supply chain and leagile supply chain to show the benefits of leagile supply chain. The results showed that shorten the length of supply chain, share the information, cooperation and production delay can effectively weaken the Bullwhip effect. Deif (2012) examined the performance of a lean cell under uncertainty using SD. The cell performance was compared under certain and uncertain external (demand) and internal (machine availability) conditions. Results showed that although lean cell was expected to be responsive to external waste, however, this was not the case under the considered uncertain conditions. Huang et al. (2012) compared between two models for a supply chain under two conditions of supply disruptions, without backup supplier, and with a contingent supplier. The retailer's total profits are also compared under these two circumstances of supply disruptions to assist the decision makers better dealing with the backup purchasing strategy. The supply chain studied only involves one retailer and two independent suppliers that are referred to as major supplier and backup supplier. Georgiadis and Michaloudis (2012) investigated the impact of dynamic disturbances in manufacturing process on the production planning and control in job-shop manufacturing by using SD. They analyzed the system response under 
different arrival patterns for customer orders and the existence of various real-time events related to customer orders and machine failures. They also assess the performance by determining the backlogged orders, WIP inventories and tardy jobs. Ali and Deif (2013) used SD to model a manufacturing system and investigated the effect of shrinking the target delivery delay (TDD) to increase the responsiveness level on the leanness level of the system.

From the aforementioned review, there are much more work related to lean manufacturing implementation and analysis than that dedicated for assessing the degree of leanness of systems. In addition, fewer work account for such assessment from a dynamic perspective. A deeper analysis of the reviewed papers focussing on lean assessment reveals that the assessment approaches focussed on metrics that are more endogenous (internal system parameters like throughput, quality WIP, etc.) rather than exogenous (external or customer-related parameters). The fewer research which included customer-related metrics to assess lean performance mainly focussed on response time to measure customer satisfaction. It is clear that with lean systems dedicated to value creation and given that customers define such value, more customer-related metrics should be used in assessing lean performance. It is also important to note that dynamic approaches are more favored in assessing lean performance of manufacturing systems than static approaches. In the reviewed literature, limited work considered the dynamic nature of assessment especially when it comes to capturing demand uncertainty. Also, the review shows that SD as a powerful dynamic modeling tool had been dedicated mainly to general manufacturing systems application or supply chain while very few work used SD in the lean assessment context.

This research contributes to the few literature available for dynamic assessment of lean systems. One contribution is through integrating service level as a customer-related measure with WIP and overall equipment effectiveness (OEE) as internal performance measures in one new metric. Furthermore, this work proposes a SD model to capture the dynamic impact of various system's parameters (including for the first time the uncertainty of demand in terms of both volume and mix) on lean performance of a manufacturing system. The new model and metric in addition to the presented analysis offers various insights and recommendations for lean management policies.

\section{Dynamic manufacturing model with leanness score}

The model introduced by Ali and Deif (2014) is modified as shown in Figure 1. The model is developed using Vensim ${ }^{\mathrm{TM}}$ as an interactive SD simulation environment. Data for a real case study in the central kitchen industry will be used to demonstrate the model, measure the degree of leanness and assess the impact of significant parameters on the new leanness score. The modeled manufacturing system consists of four components; production system, backlog system, quality system and leanness score calculation system. The production system includes five stages, three of them are dedicated for assembly and/or manufacturing, one for finishing processes, and the final one for finished goods inventory which is highly linked to the quality inspection rate. All stages are controlled by stochastic cycle time at each stage. The different model components (stages) are explained in details in the following sections.

\subsection{Model components}

3.1.1 Production system. A multi-stage production system representing several production activities is used to model the manufacturing system as shown in Figure 2. The developed multi-stage, multi-product production system is a WIP-based system 


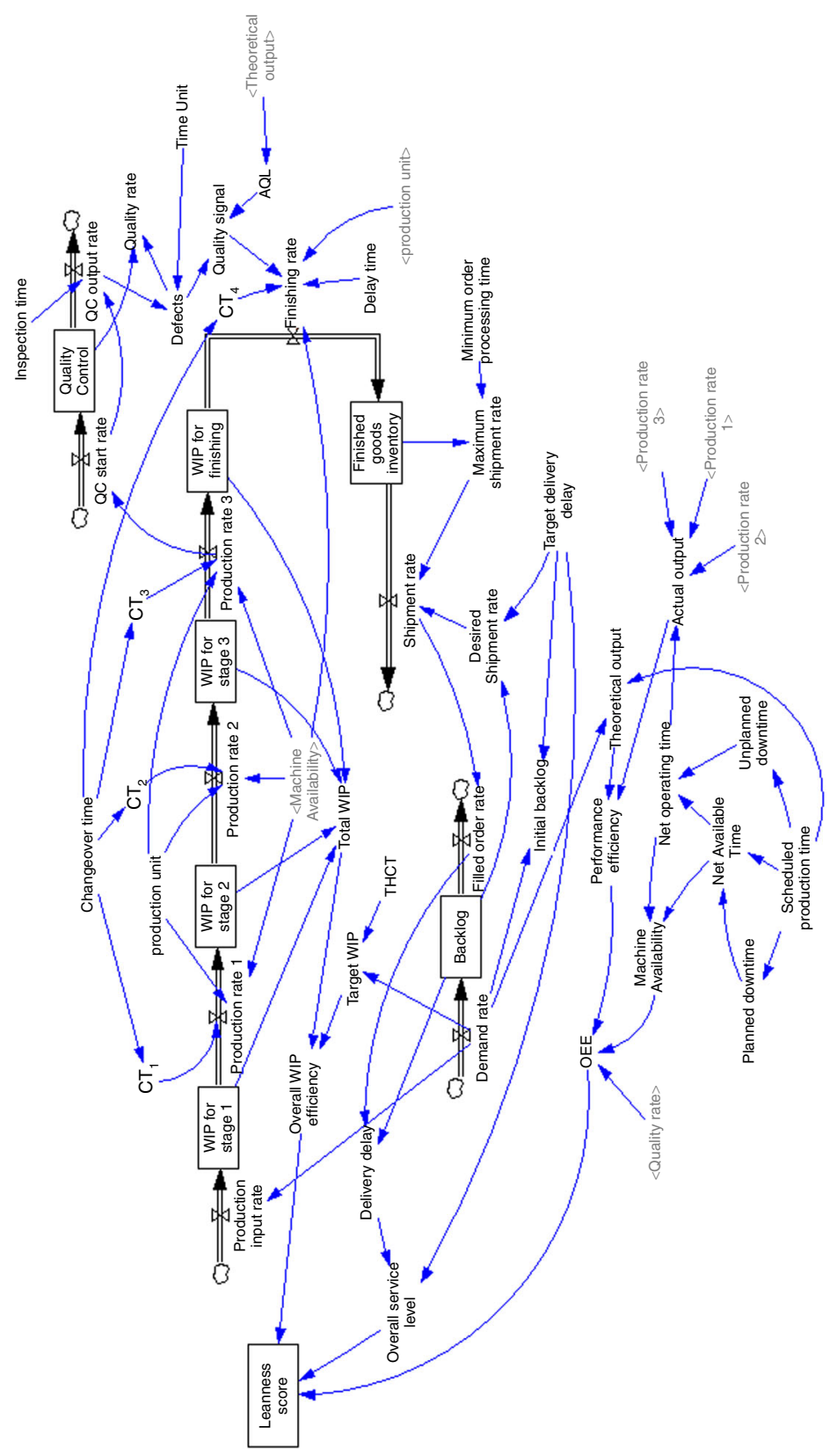

Figure 1.

Dynamic manufacturing model 
where the WIP level is controlled by changing the production rate as shown in Equation (1). The WIP level at each station is calculated by the difference between the production rate of the current station and the production rate of the subsequent one:

$$
\mathrm{WIP}_{i}(t)=\operatorname{INTEG}\left(\operatorname{IF~THEN~ELSE~}\left(\mathrm{PR}_{i}{ }_{1}(t)-\mathrm{PR}_{i}(t) \geqslant 0, \mathrm{PR}_{i}(t)-\mathrm{PR}_{i}(t), 0\right), 0\right)
$$

Production rate is expressed as the reciprocal of cycle time as seen in Equation (2). The production rates of each station (with the exception of the production input rate) are affected by the availability of the machines and their cycle times. For simplicity, it is assumed that all stations inside the production system have the same availability. This assumption will not affect the analysis in this case. As for the cycle times they are modeled using stochastic random normal distribution functions as shown in Equation (3). Normal distribution is an acceptable reflection to the uncertainty associated with typical machine times in the considered case study:

$$
\begin{gathered}
\operatorname{PR}_{i}(t)=\frac{\text { production unit }}{\mathrm{CT}_{i}(t)} \frac{\mathrm{MA}(t)}{100} \\
\mathrm{CT}_{i}(t)=\text { RANDOM NORMAL (Min, Max, Mean, SD, Seed) } \operatorname{COT}(t)
\end{gathered}
$$

The input production rate is equal to the demand rate (no over capacity is considered) as this reflects the traditional practice of lean systems trying to produce at demand (see following equation):

$$
\operatorname{PIR}(t)=\operatorname{DR}(t)
$$

Since the manufacturing system is producing products in the same family, it is assumed that changeover time is constant for all stages and it is also modeled using normal distribution to capture more of the uncertainty in the considered manufacturing environment (see following equation):

$$
\operatorname{COT}(t)=\text { RANDOM NORMAL (Min, Max, Mean, SD, Seed) }
$$

3.1.2 Backlog system. Order backlog measures the delay between the placement and delivery of orders (see Figure 3 ). Such delays can be caused by administrative activities

Figure 2.

Production system

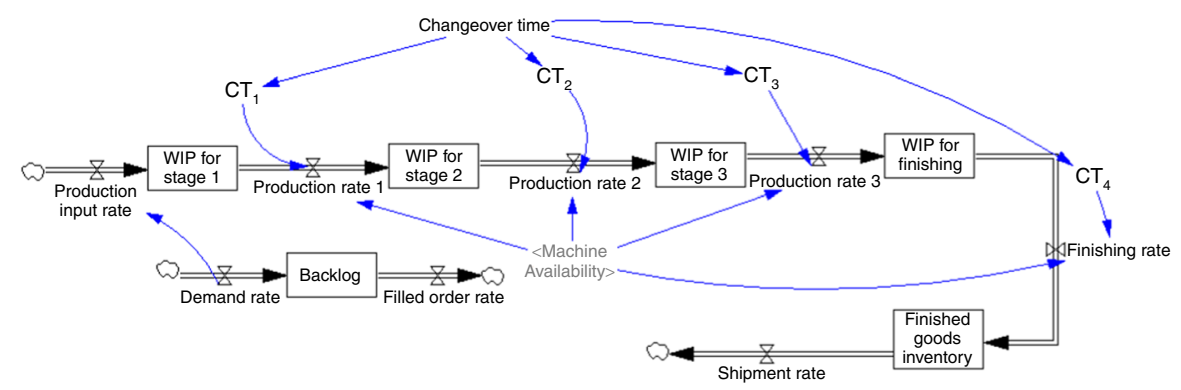


such as credit approval and order processing, by the need to customize or configure the product to the needs of particular customers, and by delays in shipping to the customer site, among others. Backlog is captured as the difference between the required demand rate and the actual filled order rate as expressed in following equation:

$$
B(t)=\operatorname{INTEG}\left(\mathrm{DR}(t)-\mathrm{FOR}(t), B_{0}\right)
$$

The initial backlog is set to equal the TDD of incoming orders (see following equation):

$$
B_{0}(t)=\mathrm{TDD} \quad \mathrm{DR}(t)
$$

While the shipment rate and filled order rate are numerically equivalent and have same units (product/hour), they are two dissimilar concepts as shown in Equation (8). The shipment rate represents the physical product that leaves the organization, while the order fulfillment rate captures the information flow (Sterman, 2000):

$$
\operatorname{FOR}(t)=\mathrm{SR}(t)
$$

The shipment rate as shown in Equation (9) depends on the minimum between either the desired shipment rate or the maximum shipment rate:

$$
\operatorname{SR}(t)=\operatorname{MIN}(\operatorname{DSR}(t), \operatorname{MSR}(t))
$$

The desired shipment rate is the rate that ensures that orders are filled within the TDD as seen in Equation (10). In a lean context, firms would strive to minimize the TDD:

$$
\operatorname{DSR}(t)=\frac{B(t)}{\mathrm{TDD}}
$$

The maximum shipment rate based on the firm's present inventory level and the minimum order processing time as specified by following equations:

$$
\operatorname{MSR}(t)=\frac{\operatorname{FGI}(t)}{\operatorname{MOPT}(t)}
$$

$$
\operatorname{FGI}(t)=\operatorname{INTEG}(\operatorname{IF~THEN~ELSE~}(\operatorname{FR}(t)-\operatorname{SR}(t) \geqslant 0, \operatorname{FR}(t)-\operatorname{SR}(t), 0), 1)
$$

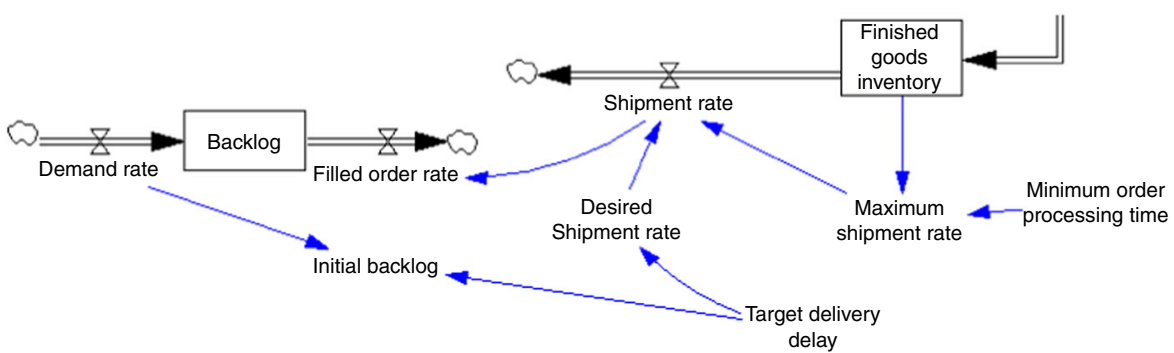

Figure 3. Backlog system 
The minimum order processing time represents the minimum time required to process and ship an order and it is expressed as normal random function as shown in following equation:

$$
\operatorname{MOPT}(t)=\text { RANDOM NORMAL (Min, Max, Mean, SD, Seed) }
$$

3.1.3 Quality system. Quality control level is calculated as the difference between the quality control output rate and quality control start rate as shown in following equation (see Figure 4):

$$
\mathrm{QC}(t)=\operatorname{INTEG}(\operatorname{IF} \operatorname{THEN} \operatorname{ELSE}(\mathrm{QCSR}(t)-\mathrm{QCOR}(t) \geqslant 0, \mathrm{QCSR}(t)-\mathrm{QCOR}(t), 0), 1)
$$

The sampling plan in this system is conducted after the third production stage where a specified number of samples are sent for quality testing as shown in following equation:

$$
\mathrm{QCSR}(t)=\mathrm{PR}_{3}(t) \quad(\text { sample size })
$$

The quality control output rate equals the quality control start rate with a fixed delay function accounting for inspection and rework times as shown in following equation:

$$
\mathrm{QCOR}(t)=\operatorname{DELAY~FIXED}(\operatorname{QCSR}(t), \operatorname{IT}(t), \operatorname{QCSR}(t))
$$

Inspection time is expressed as random normal function to reflect the stochastic nature of the inspection process as shown in following equation:

$$
\text { IT }(t)=\text { RANDOM NORMAL (Min, Max, Mean, SD, Seed) }
$$

The inspection process is designed based on the acceptable quality level (AQL) and directly controls the finishing rate. If the number of defects is less than the AQL, the finishing rate is delayed by only the inspection cycle time. However, if the number of

Figure 4.

Quality system

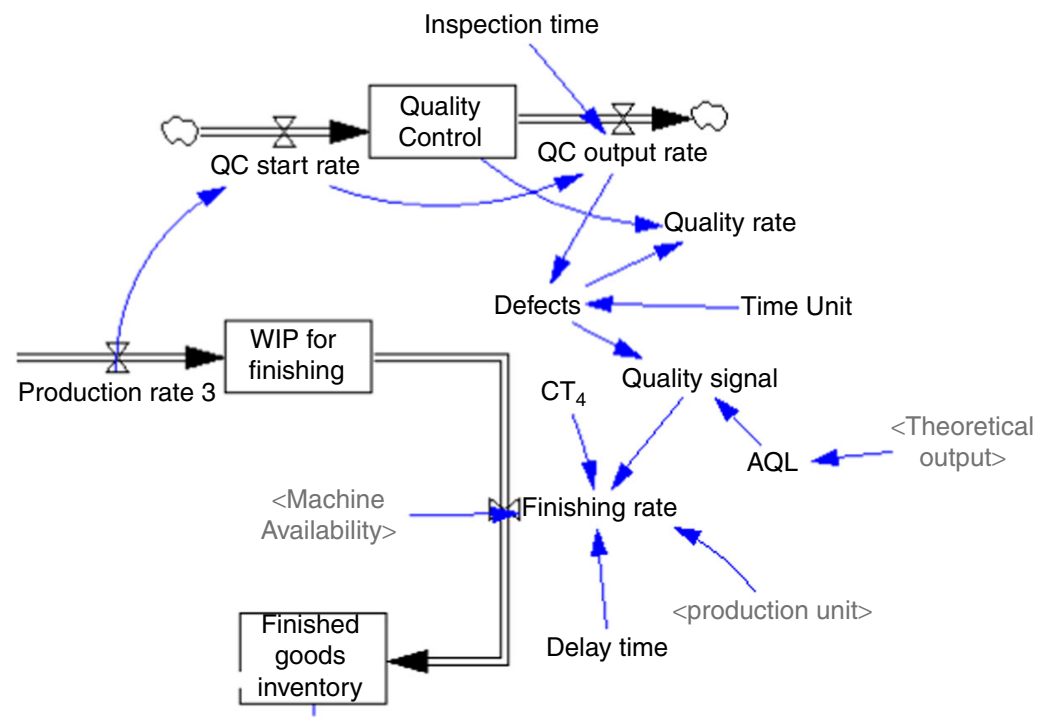


defects is greater than AQL, the finishing rate is delayed by inspection time and an extra rework delay time as indicated in following equations:

$$
\begin{gathered}
\mathrm{FR}(t)=\mathrm{IF} \text { THEN ELSE } \mathrm{QS} \geqslant 1, \frac{\text { Production unit }}{\mathrm{CT}_{4}(t)}, \frac{\text { Production unit }}{\mathrm{CT}_{4}(t)+\mathrm{DT}} \frac{\mathrm{MA}(t)}{100} \\
\mathrm{QS}(t)=\mathrm{IF} \operatorname{THEN} \operatorname{ELSE}(D(t) \leqslant \mathrm{AQL}, 1,0)
\end{gathered}
$$

The number of defects is displayed in Equation (20) as a stochastic function of quality control output rate:

$$
\begin{aligned}
\mathrm{D}(t)= & \text { RANDOM NORMAL }(\text { Min QCOR, Max QCOR, Mean } \\
& \text { QCOR, SD QCOR, Seed }) \times \text { Time Unit }
\end{aligned}
$$

3.1.4 Leanness score system. The new leanness score system developed in this model consists of three metrics (shown in Equation (21)) which are: overall work-in-process efficiency (OWE), OEE and overall service level (OSL) as displayed in Figure 5.

The new assessment system is meant to capture three fundamental lean outcomes in any system, mainly production stability/leveling (reflected in the WIP level), production efficiency (reflected in quality and availability) and responsiveness to market (reflected in service level). The leanness score is presented as percentage ensures that the assessment is dimensionless for tracking and comparison purposes. In this paper, the score is the average of the three components and all the three components have the same weight which can be changed in further research (where some lean results could be of concern to the manufacturing mangers and thus would receive higher weight). The three components of the proposed leanness score are explained as follows:

$$
\mathrm{LS}(t)=\frac{\mathrm{OEE}(t)+\mathrm{OWE}(t)+\mathrm{OSL}(t)}{3} \quad 100
$$

3.1.4.1 Overall WIP efficiency. OWE is an indicator of the accumulation of WIP overtime reflecting internal efficiency as well as stability (see Figure 6). A manufacturing unit must balance WIP to maintain productivity for known and future requirements. So, organizations should establish its target WIP and compare their actual WIP with this target as shown in following equation:

$$
\operatorname{OWE}(t)=\operatorname{IF} \operatorname{THEN~ELSE} \quad \operatorname{TGWIP}(t) \leqslant \operatorname{TWIP}(t), \frac{\operatorname{TGWIP}(t)}{\operatorname{TWIP}(t)}, \frac{\operatorname{TWIP}(t)}{\operatorname{TGWIP}(t)}
$$

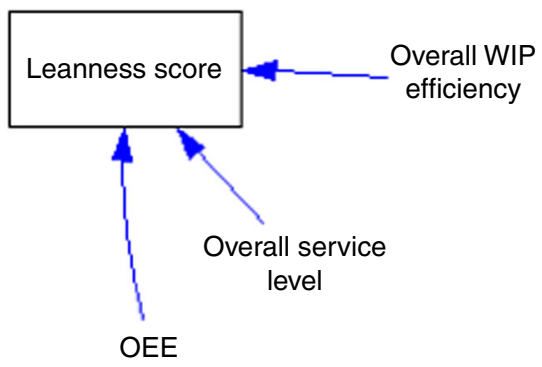

Figure 5. Leanness score system 
Target WIP level is calculated based on little's law multiplying the demand rate (assuming that in the ideal lean state it should equate the production rate) by the theoretical cycle time. Actual WIP level is the average of all WIP accumulated over the different production stages (see following equations):

$$
\begin{aligned}
\operatorname{TGWIP}(t) & =\operatorname{DR}(t) \quad \operatorname{THCT} \\
\operatorname{TWIP}(t) & =\frac{\sum_{i=1}^{4} \mathrm{WIP}_{i}(t)}{4}
\end{aligned}
$$

3.1.4.2 OEE. OEE is a measure for lean manufacturing environments, and it should be viewed as a "Continuous Improvement Engine" that provides a robust outline for the lean journey (see Figure 7). OEE consists of three factors: performance efficiency, machine availability and quality rate. It can be calculated as displayed in following equation:

$$
\mathrm{OEE}(t)=\frac{\mathrm{MA}(t) \quad \mathrm{PE}(t) \quad \mathrm{R}(t)}{100100100}
$$

Figure 6.

Overall WIP efficiency
Figure 7.

Overall equipment effectiveness
The availability portion of the OEE metric represents the percentage of scheduled time that the operation is available to operate and it is calculated as Equation (26). The availability metric is a pure measurement of uptime that is designed to exclude the
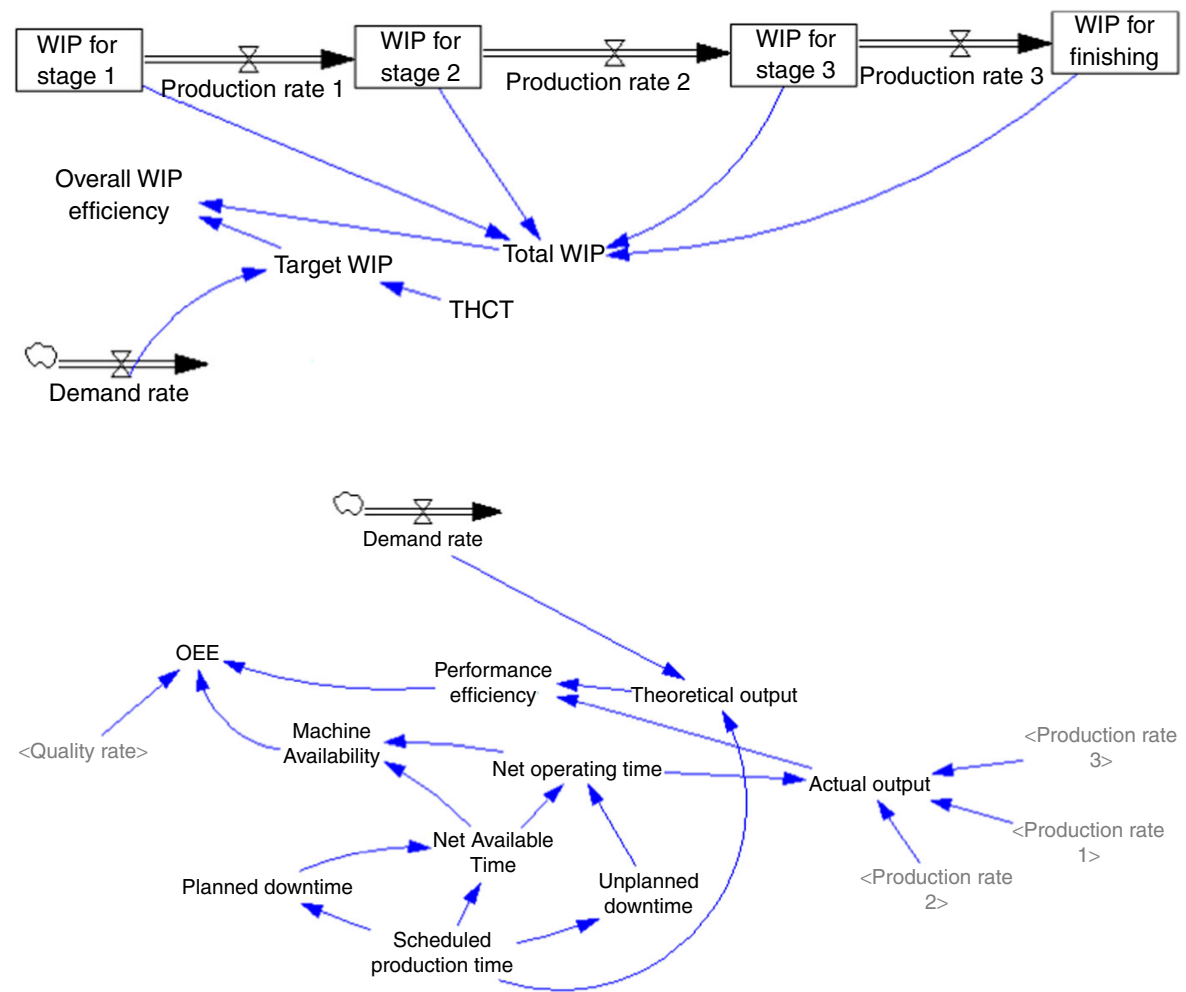
effects of quality, performance and scheduled downtime events. The losses due to wasted availability are called availability losses:

$$
\operatorname{MA}(t)=\frac{\operatorname{NOT}(t)}{\mathrm{NAT}} \quad 100
$$

Net available time is calculated as the scheduled production time subtracted by planned downtime (see following equation):

$$
\mathrm{NAT}=\mathrm{SPT} \text { PDT }
$$

Net operating time is the unplanned downtime subtracted from net available time (see Equation (28)). Unplanned downtime is the unscheduled downtime events and it is calculated as a random function to reflect the stochastic uncertainty of machines in production environments as shown in Equation (29):

$$
\begin{gathered}
\operatorname{NOT}(t)=\operatorname{NAT} \operatorname{UPDT}(t) \\
\operatorname{UPDT}(t)=\text { RANDOM NORMAL (Min, Max, Mean, SD, Seed) SPT }
\end{gathered}
$$

The performance portion of the OEE metric represents the speed at which the work center runs as a percentage of its designed speed. The performance metric is a pure measurement of speed that is designed to exclude the effects of quality and availability. The losses due to wasted performance are also often called speed losses. It is calculated as the percentage between actual output and theoretical output (see Equation (30)). Deviation from target output indicates cases of either lower production or overproduction and both are considered wastes from lean perspective:

$$
\begin{aligned}
\operatorname{PE}(t)= & \operatorname{IF} \text { THEN ELSE } \quad \operatorname{AOUT}(t) \geqslant \operatorname{ThOUT}(t), \quad \frac{\operatorname{THOUT}(t)}{\operatorname{AOUT}(t)} \\
& 100, \frac{\operatorname{AOUT}(t)}{\operatorname{THOUT}(t)} \quad 100
\end{aligned}
$$

Actual output is the net operating time times the minimum of all the three production stages rates as shown in following equation:

$$
\operatorname{AOUT}(t)=\operatorname{NOT}(t) \quad \operatorname{MIN}\left(\operatorname{MIN}\left(\mathrm{PR}_{3}(t), \mathrm{PR}_{2}(t)\right), \mathrm{PR}_{1}(t)\right)
$$

Theoretical (or target) output is the multiplication of scheduled production time and the demand rate (see following equation):

$$
\operatorname{THOUT}(t)=\mathrm{SPT} \quad \mathrm{DR}(t)
$$

The quality portion of the OEE metric represents the good units produced as a percentage of the total units produced (see Equation (33)). The quality metric is a pure 
measurement of process yield that is designed to exclude the effects of availability and performance. The losses due to defects and rework are called quality losses:

$$
\mathrm{QR}(t)=\frac{\mathrm{QC}(t) \mathrm{D}(t)}{\mathrm{QC}(t)} \quad 100
$$

3.1.4.3 OSL. OSL reflects the level at which the customer orders is filled on time (see Figure 8). It is the ratio between the TDD and actual delivery delay as shown in Equation (34). The actual delivery delay is the ratio between the backlog in the system and the filled order rate as seen in Equation (35):

$$
\begin{aligned}
& \operatorname{OSL}(t)=\frac{\mathrm{TDD}}{\mathrm{DD}(t)} \\
& \mathrm{DD}(t)=\frac{\mathrm{B}(t)}{\mathrm{FOR}(t)}
\end{aligned}
$$

\section{Significance of demand dynamics on leanness level}

The analysis in this paper is based on the data of an industrial case study of a kitchen equipment manufacturer. The company is one of the leading companies in Egypt in the field of central kitchen equipment since 1980. Their business scope is importing, designing, manufacturing and supplying all central kitchens equipment and laundries for hotels, touristic villages, restaurants and hospitals. The company's data used by the developed model is shown in Table AI.

Before investigating the impact of demand dynamics on the developed leanness score, the significance of demand among other parameters in the presented model was explored. A DOE technique was employed for that purpose where the six stochastic parameters of the 15 input parameters where selected for the DOE analysis. Excluding the deterministic parameters was based on our interest to explore the dynamics generated by uncertainty and variability. The six parameters are shown in Table I.

A full factorial DOE method was implemented where each factor had two levels selected as a minimum and maximum. The values for each of the parameters were based on the selected case study historical data. The $2^{6}=64$ runs were carried out while monitoring the response (leanness score). Full factorial was used to identify main effects and factor interactions without any confounding or ambiguity (Montgomery, 2005). All runs and their response are shown in Table AII.

The significance of main factors and the two factors interactions were investigated. The normal plot for standardized effects is shown in Figure 9.

Figure 8.

Overall service level

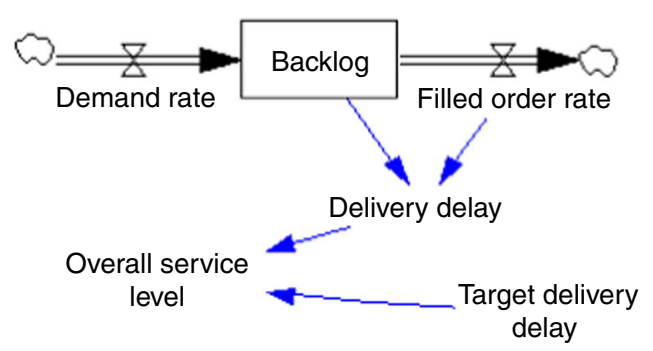


From Figure 2, the most significant factors are demand rate (highest), defects, changeover time with demand rate interaction, demand rate with unplanned downtime interaction, defects with demand rate interaction, unplanned downtime, minimum order processing time, changeover time and they are shown in Figure 10 with their relative significance.

It is shown from these results that demand rate has a major effect on the leanness score. The significance DOE analysis was used to confirm and pave the road to the importance of exploring the impact of demand dynamics on the leanness level of manufacturing systems. Understanding demand variability and its impact on leanness level and how it relates to other internal parameters is vital in successful lean implementation and this what will be partially examined in the next sections.

\section{Impact of demand dynamics on leanness score}

After examining the parameters' significance and illustrating that the most significant parameter on leanness score in the developed model is demand rate, in this section, the leanness score and its components are investigated under different degrees of demand variability. Demand variability is captured using coefficient of variation (CV). CV refers to a statistical measure of the distribution of data points in a data series around the mean.

\begin{tabular}{|c|c|c|c|}
\hline Parameter & Minimum & Maximum & \\
\hline COT & 0.003 & 0.005 & \\
\hline$D$ & $(5 / 100) \times \mathrm{QCOR}$ & $(10 / 100) \times \mathrm{QCOR}$ & \\
\hline DR & 10 & 20 & \\
\hline IT & 0.005 & 0.007 & \\
\hline MOPT & 6 & 7 & Table I. \\
\hline UPDT & $0.016 \times \mathrm{SPT}$ & $0.03 \times \mathrm{SPT}$ & Parameters values \\
\hline
\end{tabular}

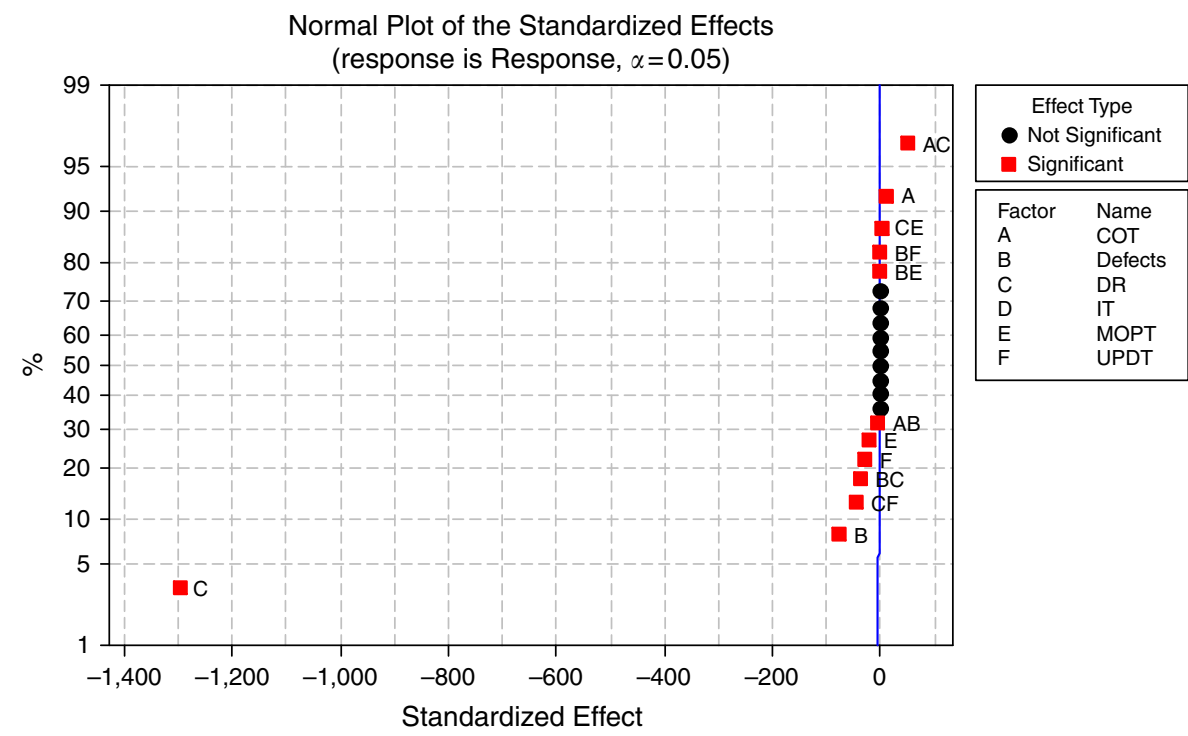

Figure 9. Significance plot 
Figure 10.

Pareto chart for significance

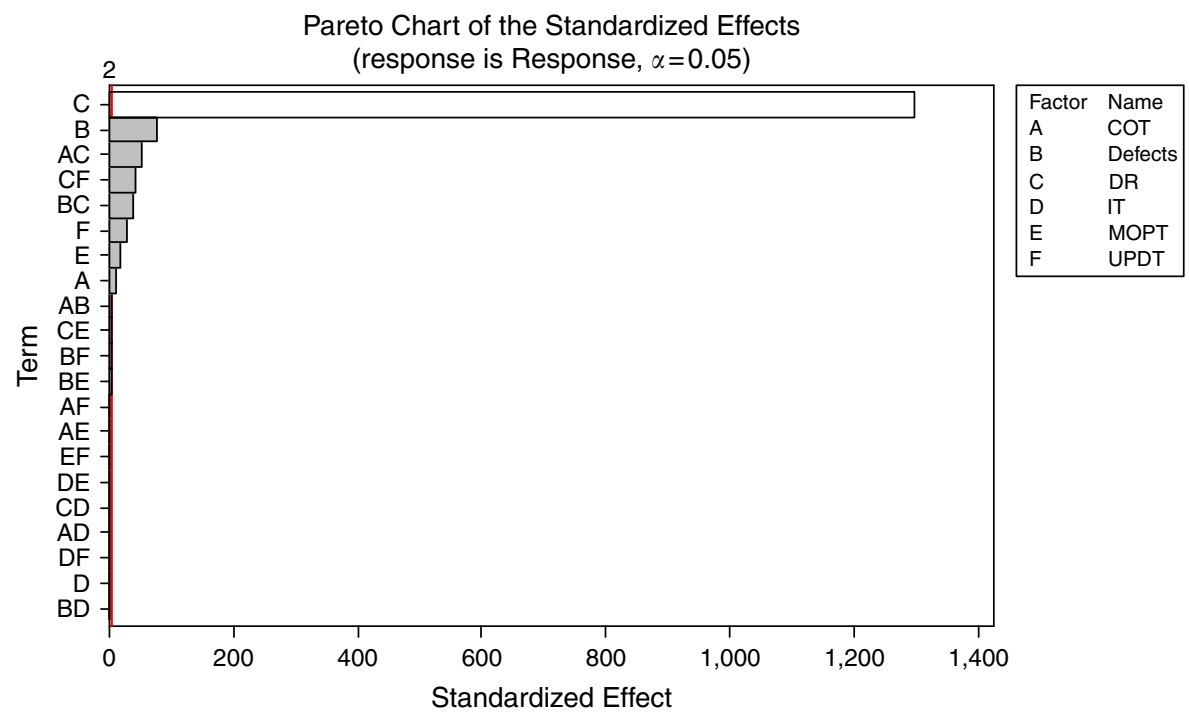

It represents the ratio of the standard deviation to the mean. The data are taken from the company history and gathered from the last 10 years.

First, the leanness score and its components' will be examined under low levels of demand variation $(\mathrm{CV}=0.33,0.5,0.22)$ with varying levels of demand means. Then, the dynamics associated with higher levels of demand variation (i.e. $\mathrm{CV}=0.5,1.5,1$ ) with constant mean will be investigated.

\subsection{Dynamics of low demand CV with varying means}

The behavior of the model is investigated in this section under overall low level of CV. However, the average and the standard deviation of the selected demand patterns are changing at different magnitudes to be able to see the behavior of the system when excited under different means of demand. Table II shows the different values of demand mean, standard deviation and their CV.

In this analysis, various demand patterns are considered. The first pattern is one of high demand average and high standard deviation (the highest $\mathrm{CV}$ ) and it is referred to as $\mathrm{DR}_{1}$, the next pattern is the low demand average and low standard deviation and it is referred to as $\mathrm{DR}_{2}$. Finally the system is investigated under what we labeled as the normal average demand referred to as DR which fits between the previous two patterns. Such analysis will enable lean practitioners to envision the impact of different demand excitation patterns to the systems and also draw a clearer picture of how demand variability in general (going from low to normal to high and also varying both average

Table II.

Data for low demand coefficient of variation with various means values

\begin{tabular}{lll}
\hline & CV & Demand rate value \\
\hline DR & 0.33 & RANDOM NORMAL $(10,20,15,5,1)$ \\
$\mathrm{DR}_{1}$ & 0.5 & RANDOM NORMAL $(21,63,42,21,1)$ \\
$\mathrm{DR}_{2}$ & 0.22 & RANDOM NORMAL $(8,12,10,2,1)$ \\
\end{tabular}


and standard deviation) can help better understand how a lean system reacts and thus develop optimal demand management policies as well as internal operational plans.

5.1.1 The impact of low demand CVS on the OEE. Figure 11 shows the effect of the considered demand patterns on the OEE.

It is shown from the figure that the highest effectiveness for equipment, in the selected settings, was maintained with the normal demand rate DR which has a medium $\mathrm{CV}$. The main reason behind this performance was that the current production level average was close to the mean of this demand pattern leading to high production efficiency. $\mathrm{DR}_{1}$ which has the highest $\mathrm{CV}$ shows the lowest OEE due it's mean high deviation from the current production rate and also due to high level of variability that impact both quality and production availability. On the other hand, $\mathrm{DR}_{2}$; which have the lowest $\mathrm{CV}$ has a relative moderate OEE performance between the previous two demand patterns. The cases of higher or lower values of demand means than the production level will have a negative effect on the OEE metric. This is because the theoretical output depends on the demand rate, so higher or lower values of demand will affect the ratio between the theoretical output and the actual output negatively and in turn the performance efficiency and OEE. The variability level (reflected in the CV values) was not the major contributor to the OEE performance.

5.1.2 The impact of low demand CVs on the OSL. The effect of the considered demand patterns on the OSL is shown in Figure 12.

It can be observed that $\mathrm{DR}_{2}$ pattern has the highest and fasted level of OSL performance, while over longer time (64 hours or eight days) the OSL for DR pattern reaches the same 100 percent level. In contrast, producing to chase the $\mathrm{DR}_{1}$ pattern will get the OSL level to decrease overtime till it reaches near zero at the end of the simulation period.

The impact of variability is clear in this analysis. Since DR pattern had a higher CV than $\mathrm{DR}_{2}$ pattern, this variation caused the backlog to accumulate till the shipment rate was increased via more production and finally reach the 100 percent service level over longer time. The system at the $\mathrm{DR}_{1}$ pattern (which has the highest value for demand mean and the highest $\mathrm{CV}$ ) showed the lowest service level performance. This manifests

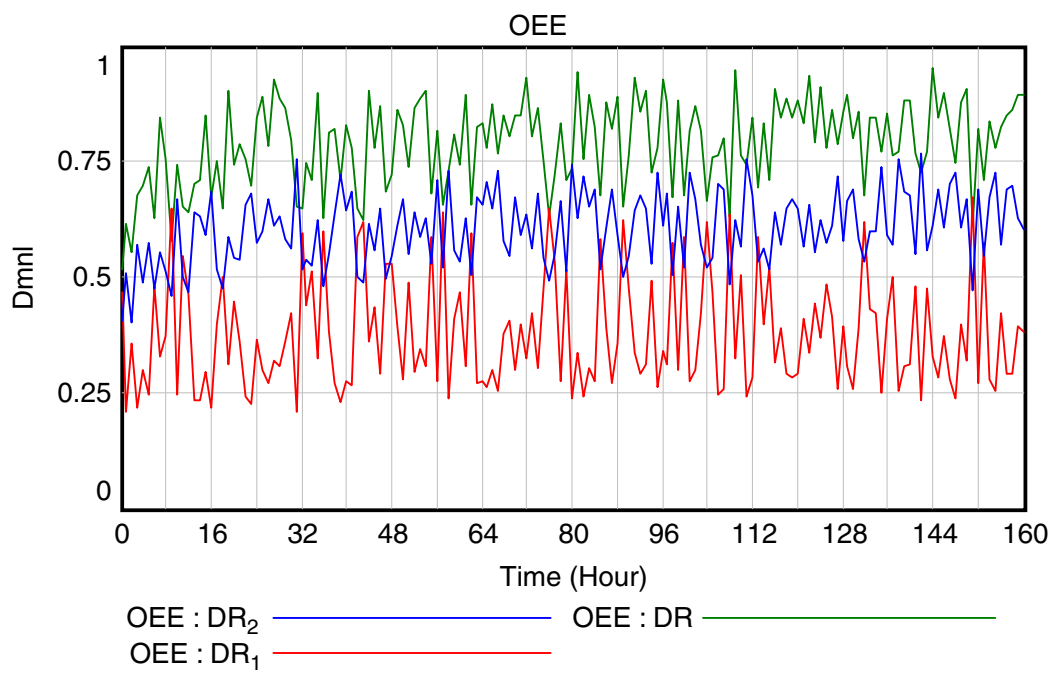

Figure 11.

OEE with low demand variation and various means 
Figure 12.

OSL with low demand variation and various means

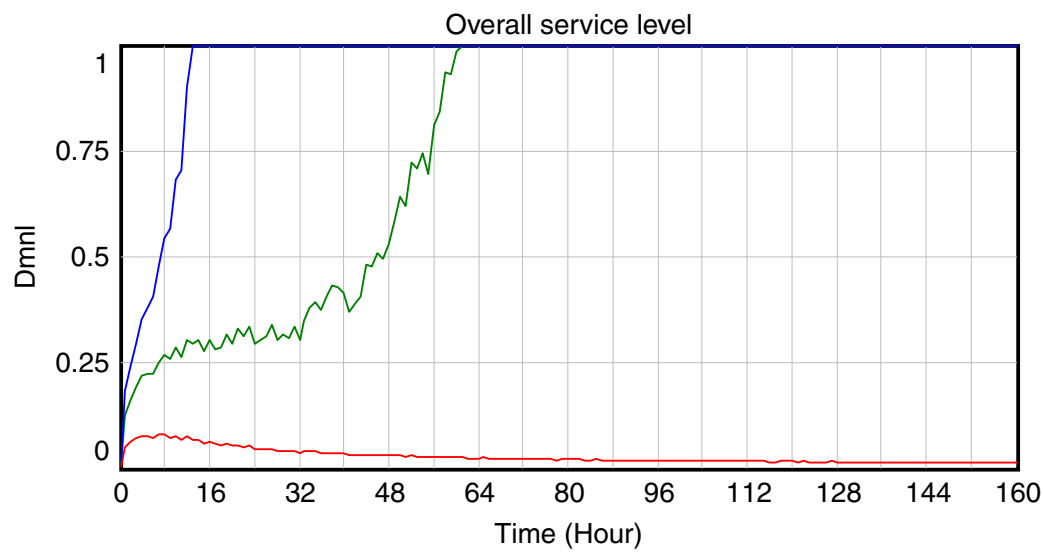

Overall service level : $\mathrm{DR}_{2}$

Overall service level : $\mathrm{DR}_{1}$

Overall service level : DR

that both the demand volume and variability contributed to the deterioration of the OSL in the considered case study. This suggests that improving service levels in these types of demand patterns requires investments and upgrades in the current production capabilities. It also suggests relaxing the TDD to be able to cope with similar demand patterns as a demand management policy.

5.1.3 The impact of low demand CVs on the overall WIP efficiency. Overall WIP efficiency behavior according to the different demand patterns is shown in Figure 13.

Since production started with zero WIP, the WIP efficiency was initially zero. After running the production, the WIP started to accumulate till it reached the desired target WIP leading to an OWE of 100 percent. However, with the considered demand patterns, the WIP continued to accumulate due to the implemented push production

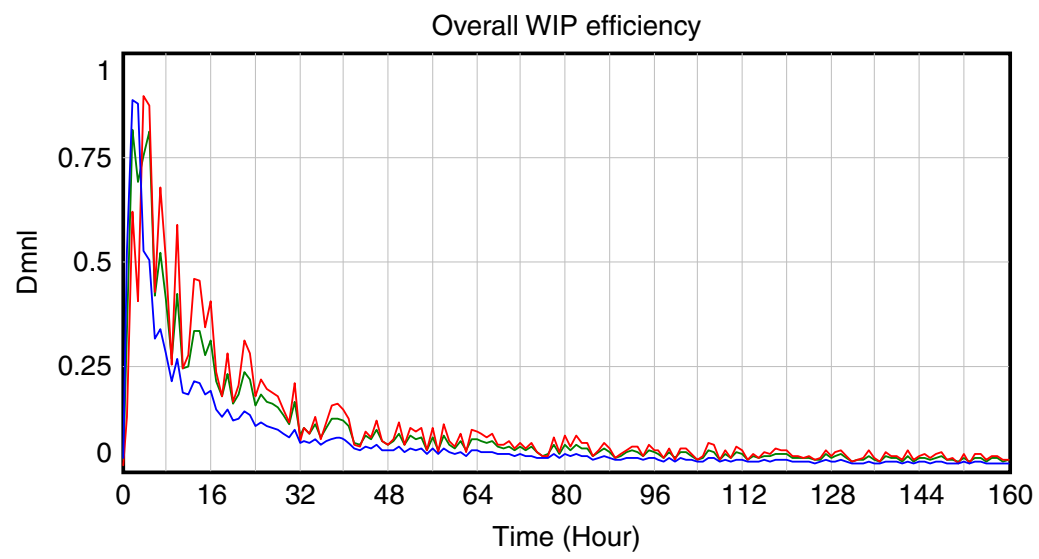

Figure 13.

OWE with low demand variation and various means
Overall WIP efficiency : $\mathrm{DR}_{2}$

Overall WIP efficiency : $\mathrm{DR}_{1}$ Overall WIP efficiency : DR 
policy and with no WIP control lean policies (like supermarkets) leading to a drop in the OWE till it reached close to zero over extended time The variability level (CV value) for each demand pattern adds to the relative speed of the deterioration of its respective OWE behavior. In these scenarios, companies have to work on smoothing the flow of production utilizing different pulling techniques from downstream.

5.1.4 The impact of low demand CVs on the leanness score. Figure 14 displays the effect of the selected demand patterns on the new developed leanness score. The figure integrates the OEE, OSL and OWE dynamic behaviors into the leanness score metric. The leanness score for the considered case study at the DR pattern (with the average demand mean and average CV) starts with a low performance then it increases till reaches the highest performance level among the three patterns (about 60 percent). Although the Leanness score for the system at $\mathrm{DR}_{2}$ pattern (lowest demand mean with lowest CV) is lower than with the DR pattern (average demand level and average CV), it shows a more steady performance than with the DR pattern due to the OSL behavior that reached its maximum level quickly. Leanness score for the system at the $\mathrm{DR}_{1}$ pattern (with the highest demand mean and highest $\mathrm{CV}$ ) starts at an average performance then decreases to less than 20 percent as the impact of its high volume and variability manifest themselves in deteriorating both the OSL as well as OWE within the system.

\subsection{Dynamics of high demand $C V$ with constant means}

The model is studied next under higher degrees of demand variability (CV) while keeping same mean for different demand patterns. Table III shows the selected demand patterns.

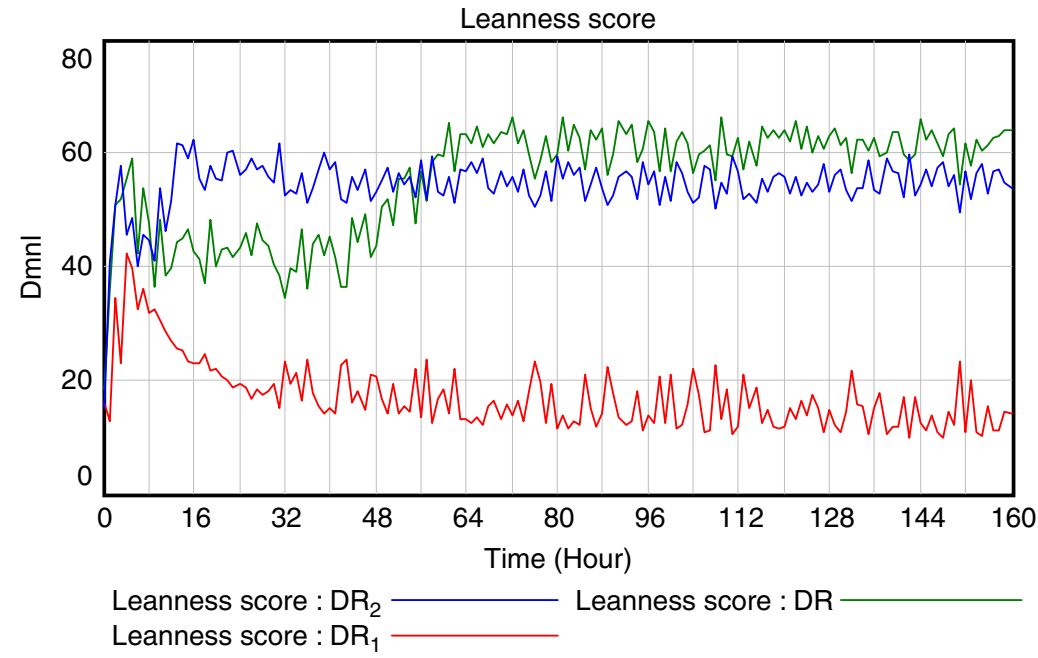

Figure 14. LS with low demand variation and various means

\begin{tabular}{lll}
\hline & CV & Demand rate value \\
\hline $\mathrm{DR}_{L}$ & 0.5 & RANDOM NORMAL $(10,20,15,7.5,1)$ \\
$\mathrm{DR}_{H}$ & 1.5 & RANDOM NORMAL $(21,63,15,22.5,1)$ \\
$\mathrm{DR}_{M}$ & 1 & RANDOM NORMAL $(8,12,15,15,1)$ \\
\hline
\end{tabular}

Table III. Data of higher demand variation with stable means scenario 
5.2.1 The impact of higher demand $C V$ on the OEE. Figure 15 shows the effect of higher CVs with stable means of demand rate on the OEE metric.

The figure shows that the system responding to the demand rate with the lowest $\mathrm{CV}$ $\left(\mathrm{DR}_{L}\right)$ has the best OEE (average of 80 percent) while responding to the demand rate with the highest $\mathrm{CV}\left(\mathrm{DR}_{H}\right)$ lead to the worst OEE performance (average of 45 percent). Although the system is responding to the same demand average, however, increasing the variability of the demand will affect negatively both the quality level and the performance efficiency leading to the deterioration of OEE performance. This aligns with the continuous effort of today's process improvement approaches (e.g. Lean Six Sigma) to capture, reduce and eliminate variations within manufacturing systems.

5.2.2 The impact of higher demand CV on the OSL. The effect of the same demand patterns on the OSL is shown in Figure 16.

The performance of the system's OSL with the $\mathrm{DR}_{M}$ demand pattern which has the medium level of variation (CV) is better than its performance with demand pattern with low CV $\left(\mathrm{DR}_{L}\right)$ which also reaches 100 percent level but after longer time. This is because within these parameter settings, the range of values for $\mathrm{DR}_{L}$ is higher than that for $\mathrm{DR}_{M}$ which caused the system to experience some higher orders in some instants leading to higher backlog in the case of $\mathrm{DR}_{L}$. These instants resemble cases like sudden increase in volume or rush orders. The system's had the worst OSL when experienced the demand with the highest CV leading to a near zero performance overtime reflecting the disability of the system to cope with this level of demand variation while maintaining the target due dates due to internal turbulence.

5.2.3 The impact of higher demand CV on the overall WIP efficiency. The behavior of overall WIP efficiency for the selected demand patterns is shown in Figure 17.

The performance of the OWE of the system with all demand patterns deteriorates overtime. This is because WIP levels usually reflects the stability of the system, and with demand patterns that exhibit variations, WIP will tend to increase in order to stabilize the system resulting in low OWE levels and leading to having the actual WIP to be higher than the target WIP. In the considered case study, the average production cycle time is

Figure 15.

OEE with higher CV and stable means of demand

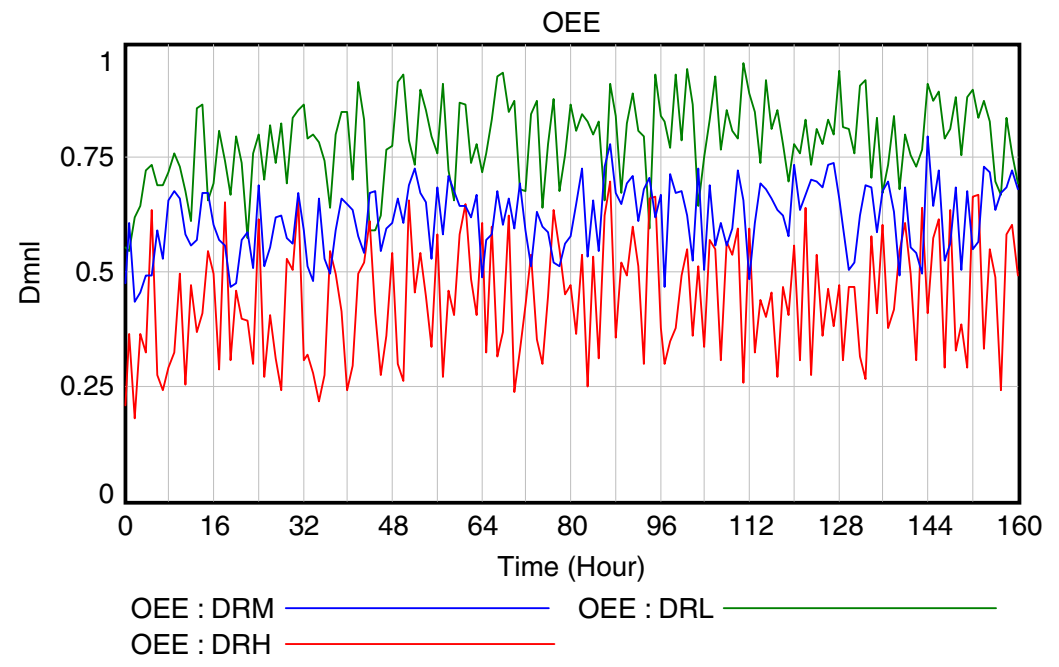




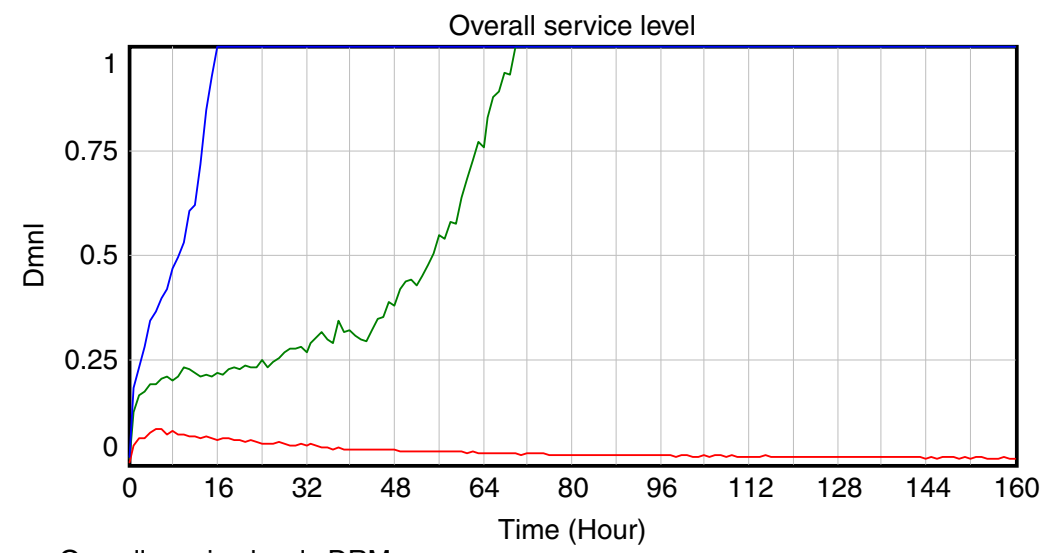

Overall service level : DRM

Overall service level : DRH

Overall service level : DRL

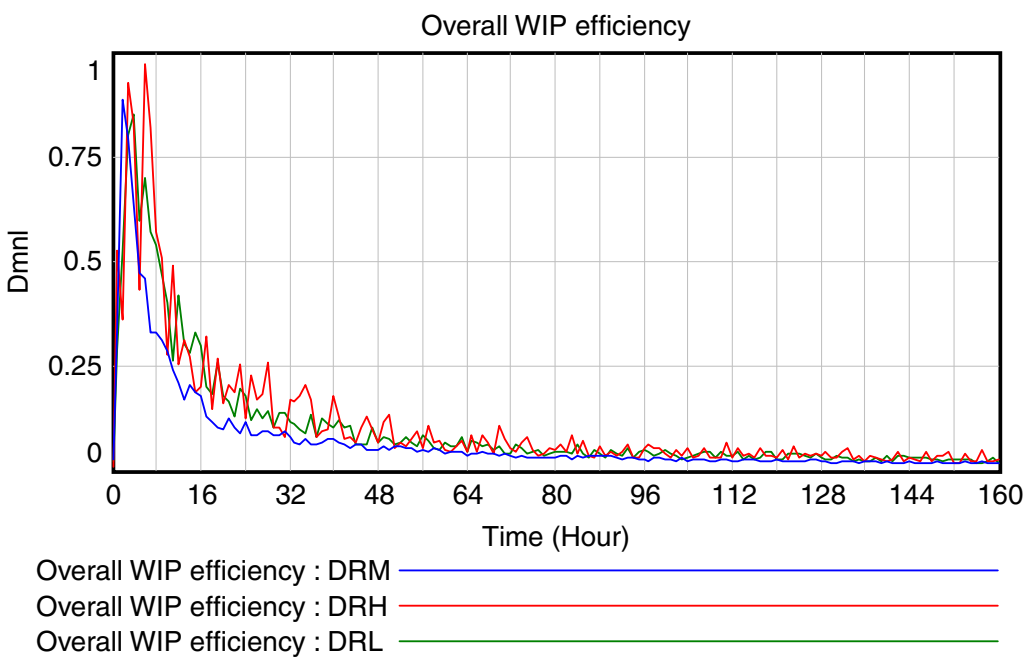

Figure 16. OSL with higher CV and stable means of demand
Figure 17.

OWE with higher $\mathrm{CV}$ and stable means of demand

close to the average demand mean of all patterns, however, the variability within these demand patterns lead to the accumulation of WIP between production stages.

5.2.4 The impact of higher demand $C V$ on the leanness score. The leanness score dynamics against the selected demand patterns is displayed in Figure 18.

The highest leanness level for the system was with the demand having the lowest variability $\left(\mathrm{DR}_{\mathrm{L}}\right)$, however, it reaches its best performance after some time due to the OSL low performance as discussed earlier. The leanness level of the system with the medium variability is close to the previous one yet with better consistency. This consistency is due to experiencing less instants of sudden increase in volume or rush orders. In this specific settings, the company is not in need to put effort to reduce the demand variation level from medium to low as it does not pay back from leanness score perspective. 
Figure 18.

LS with higher CV and stable means of demand

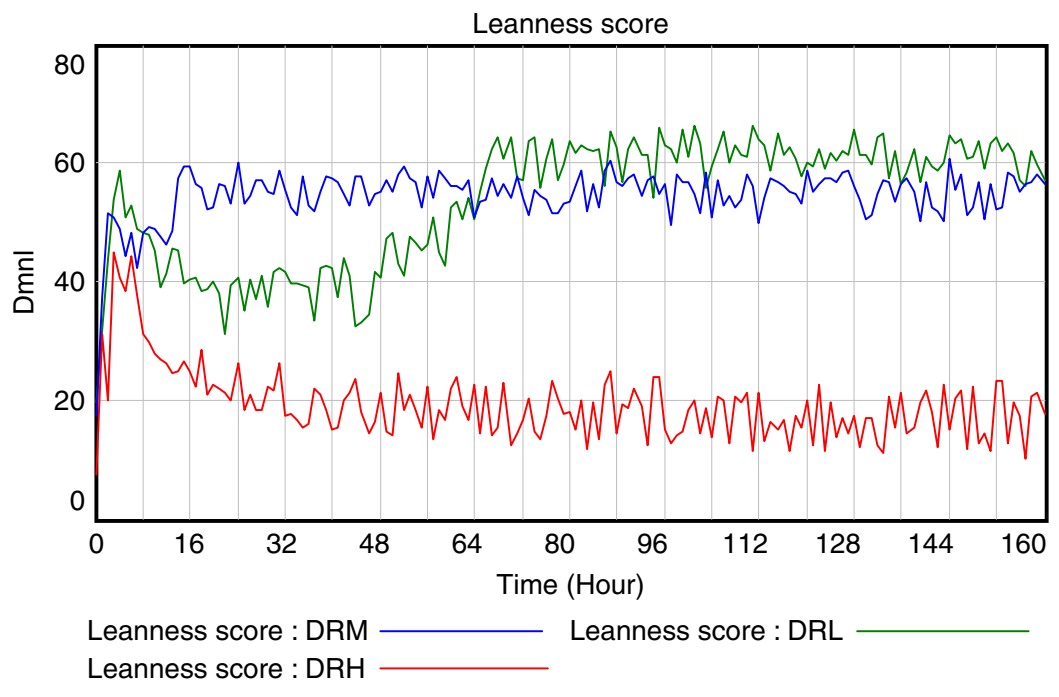

It is also clear that the system when experiencing the highest variation with the $\mathrm{DR}_{H}$ pattern will have the worst leanness profile. In such demand scenarios, decision makers should pursue more effort in demand management to avoid or reduce this level of variation or adjust the system capabilities to cope better with such levels.

\section{Summary and recommendations}

This paper presented a new metric to assess the degree of leanness of a manufacturing system under dynamic conditions and was further demonstrated using a real case study. The new metric integrated OEE, service level and WIP performance to measure the system's leanness degree. This integration offers a more comprehensive leanness assessment that reflects system efficiency, responsiveness to customers and internal stability. Such integration is critical within lean systems that focus on value creation to customers as well as internal system performance. Furthermore, the model captured the dynamics associated with different manufacturing uncertainties and their relation to the developed leanness score (including for the demand volume and variability dynamics).

A DOE analysis was carried out to examine the most significant parameters affecting the proposed leanness score which was shown, given the selected input data of the case study, to be the demand rate. This was followed by an analysis to examine the impact of demand volume and variability on the leanness level and its components. The following lean management policies and recommendations (within the scope of similar manufacturing systems to the considered company) were obtained:

- The OEE dynamics was negatively affected in all demand scenarios by variability. This is associated to the adverse effect variation has on quality and productivity. However, it was shown that in cases of low variation (lower values of CV), the higher impact on OEE was the synchronization between demand mean and the current production level average. The results suggest that to improve OEE, demand management policies should reduce variability passed on from the demand patterns to production and that production planners should work to align production levels with the demand mean to avoid lower productivity results. 
- The service level in the presented model was sensitive to high level of variation in demand showing very low performance with high CV. As variability is reduced, service level increases overtime depending on the production ability to catch up and clear backlog. In addition, instants of sudden increase in volume and rush orders contributed to the reduction in the OSL performance in the considered case study. The responsiveness level in dynamic demand environment can be improved (in addition to the typical variation reduction recommendation) by maintaining a dynamic production capacity that can scale up productivity with fast ramp up profile to clear backlog accumulated due to demand variability.

- The overall WIP efficiency in the presented analysis demonstrated how demand variation at any level will lead to high levels of accumulated WIP. WIP accumulation is an expected internal production reaction in order to maintain stability and efficiency. This phenomenon was further manifested due to the push production policies employed by the selected case study. Results confirm the importance of absorbing demand variation through different lean techniques like a balanced Heijunka boxes as well as moving toward continuous flow if possible or at least employing different pull techniques to avoid high level of WIP accumulations.

- The new leanness score was demonstrated to be a successful assessment metric for the system's leanness level in this dynamic environment. Results highlighted how the metric reflected the general deterioration trend of the leanness level of the system under high level of variations even with smaller volumes of demand. In addition, the new score was useful in showing how the leanness level of the system using the three considered components (and given the selected case study) is very close in cases of low and medium variations. This can help lean mangers in deciding whether the investment in managing demand to reduce the level of variation from medium to low and/or to increase production capacity will pay off or not.

The presented work will be extended to include more industrial applications. In addition, the impact of stochastic changeover time and inspection time on the leanness score performance will be investigated. Furthermore, the economic perspective of lean implantation will be studied by adding the cost element into the proposed model and metric. Finally, changing the weight for each of the leanness score components will be studied to reflect different managerial policies and priorities.

\section{References}

Abdulmalek, F. and Rajgopal, J. (2007), "Analyzing the benefits of lean manufacturing and value stream mapping via simulation: a process sector case study", International Journal of Production Economics, Vol. 107 No. 1, pp. 223-236.

Ali, R. and Deif, A. (2013), "Investigating the impact of the dynamics associated with increasing responsiveness level on leanness", Proceedings of Industrial and Systems Engineering Research Conference, Puerto Rico.

Ali, R. and Deif, A. (2014), "Dynamic lean assessment for takt time implementation", Procedia CIRP, Vol. 47 No. 1, pp. 2212-8271.

Almomani, M., Abdelhadi, A., Mumani, A., Momani, A. and Aladeemy, M. (2014), “A proposed integrated model of lean assessment and analytical hierarchy process for a dynamic road map of lean implementation”, International Journal of Advanced Manufacturing Technology, Vol. 72 No. 1, pp 161-172. 
Behrouzi, F. and Wong, K. (2011), "Lean performance evaluation of manufacturing systems: a dynamic and innovative approach", Procedia Computer Science, Vol. 3 No. 1, pp. 388-395.

Black, J. (2007), "Design rules for implementing the Toyota production system", International Journal of Production Research, Vol. 45 No. 16, pp. 3639-3664.

Christopher, M. (2000), "The agile supply chain competing in volatile markets", Industrial Marketing Management Journal, Vol. 29 No. 1, pp. 37-44.

Deif, A. (2012), "Dynamic analysis of a lean cell under uncertainty", International Journal of Production Research, Vol. 50 No. 4, pp. 1127-1139.

Deif, A. and ElMaraghy, H. (2014), "Cost performance dynamics in lean production leveling", Journal of Manufacturing Systems, Vol. 33 No. 4, pp. 613-623.

Detty, R. and Yingling, J. (2000), "Quantifying benefits of conversion to lean manufacturing with discrete event simulation: a case study", International Journal of Production Research, Vol. 38 No. 2, pp. 429-445.

Elnadi, M. and Shehab, E. (2014), "A conceptual model for evaluating product-service systems leanness in UK manufacturing companies”, Procedia CIRP, Vol. 22 No. 1, pp. 281-286.

Elsayed, N., Jondral, A., Greinacher, S., Dornfeld, D. and Lanza, G. (2013), “Assessment of lean and green strategies by simulation of manufacturing systems in discrete production environments", CIRP Annals - Manufacturing Technology, Vol. 62 No. 1, pp. 475-478.

Forrester, J.W. (1961), Industrial Dynamics, MIT Press, Cambridge, MA.

Georgiadis, P. and Michaloudis, C. (2012), "Real-time production planning and control system for job-shop manufacturing: a system dynamics analysis”, European Journal of Operational Research, Vol. 216 No. 1, pp. 94-104.

Helo, P. (2000), "Dynamic modeling of surge effect and capacity limitation in supply chains", International Journal Production Research, Vol. 38 No. 17, pp. 4521-4533.

Huang, M., Yang, M., Zhang, Y. and Liu, B. (2012), "System dynamics modeling-based study of contingent sourcing under supply disruptions", Systems Engineering Procedia, Vol. 4 No. 1, pp. 290-297.

Montgomery, D. (2005), Introduction to Statistical Quality Control, John Wiley \& Sons, NJ.

Pakdil, F. and Leonard, K. (2014), "Criteria for a lean organization: development of a lean assessment tool", International Journal of Production Research, Vol. 52 No. 15, pp. 4587-4607.

Poles, R. (2013), "System dynamics modelling of a production and inventory system for remanufacturing to evaluate system improvement strategies", International Journal of Production Economics, Vol. 144 No. 1, pp. 189-199.

Sanchez, A. and Perez, M. (2001), "Lean indicators and manufacturing strategies", International Journal of Operations \& Production Management, Vol. 21 No. 11, pp. 1433-1451.

Savsar, M. and Al-Jawini, A. (1995), "Simulation analysis of just-in-time production systems", International Journal of Production Economics, Vol. 42 No. 1, pp. 67-78.

Staats, B., Brunner, D. and Upton, D. (2011), "Lean principles, learning, and knowledge work: evidence from a software services provider”, Journal of Operations Management, Vol. 29 No. 5, pp. 376-390.

Sterman, J. (2000), Business Dynamic - Systems Thinking And Modeling For a Complex World, McGraw- Hill, New York, NY.

Susilawati, A., Tan, J., Bell, D. and Sarwar, M. (2015), "Fuzzy logic based method to measure degree of lean activity in manufacturing industry", Journal of Manufacturing Systems, Vol. 34 No. 1, pp. 1-11.

Taj, S. (2008), "Lean manufacturing performance in China: assessment of 65 manufacturing plants”, Journal of Manufacturing Technology Management, Vol. 19 No. 2, pp. 217-234. 
Wan, H., Chen, F. and Rivera, L. (2007), "Leanness score of value stream maps”, Proceedings of the Industrial Engineering Research Conference, Nashville, Tennessee.

Wikner, J., Towill, D. and Naim, M. (1991), "Smoothing supply chain dynamics", International Journal of Production Economy, Vol. 22 No. 3, pp. 231-248.

Womack, J. and Jones, D. (2003), Lean Thinking, Free Press, New York, NY.

Yang, T., Kuo, Y., Su, C. and Hou, C. (2015), "Lean production system design for fishing net manufacturing using lean principles and simulation optimization", Journal of Manufacturing Systems, Vol. 34 No. 1, pp. 66-73.

Zhang, Y., Wang, Y. and Wu, L. (2012), "Research on demand-driven leagile supply chain operation model: a simulation based on anylogic in system engineering", Systems Engineering Procedia, Vol. 3 No. 1, pp. 249-258.

\section{Further reading}

Alireza, A., Zulkifli, N., Yusuff, R., Hojjati, S. and Ismail, Y. (2011), "A proposed dynamic model for a lean roadmap”, African Journal of Business Management, Vol. 5 No. 16, pp. 6727-6737.

Womack, J., Jones, D. and Roos, D. (1990), The Machine that Changed the World, New York, Free Press, NY.

\section{Appendix 1}

\begin{tabular}{lll}
\hline Parameter & Value & Unit \\
\hline AQL & $(1 / 100) \times$ THOUT & Parts \\
COT & RANDOM NORMAL $(0.003,0.005,0.004,0.001,1)$ & Hour \\
$\mathrm{CT}_{1}$ & RANDOM NORMAL $(0.05,0.06,0.055,0.005,1)$-COT & Hour \\
$\mathrm{CT}_{2}$ & RANDOM NORMAL $(0.03,0.04,0.035,0.005,1)$-COT & Hour \\
$\mathrm{CT}_{3}$ & RANDOM NORMAL $(0.06,0.07,0.065,0.005,1)$-COT & Hour \\
$\mathrm{CT}_{4}$ & RANDOM NORMAL $(0.055,0.065,0.06,0.005,1)$-COT & Hour \\
$D$ & RANDOM NORMAL $(5 / 100) \times \mathrm{QCOR},(10 / 100) \times \mathrm{QCOR},(7.5 / 100) \times \mathrm{QCOR}$, & Parts \\
& $(2.5 / 100) \times$ QCOR, 1$) \times$ Time Unit & \\
$\mathrm{DT}$ & 0.005 & Hour \\
DR & RANDOM NORMAL $(10,20,15,5,1)$ & Parts/hour \\
IT & RANDOM NORMAL $(0.005,0.007,0.006,0.001,1)$ & Hour \\
MOPT & RANDOM NORMAL $(6,7,6.5,0.5,1)$ & Hour \\
PDT & $(2 / 100) \times$ SPT & Hour \\
QCSR & PR $\times(20 / 100)$ & Parts/hour \\
TDD & 2 & Hour \\
THCT & 1 & Hour \\
UPDT & RANDOM NORMAL $(0.016,0.03,0.023,0.007,1) \times$ SPT & Hour \\
\end{tabular}




\section{Appendix 2}

\begin{tabular}{|c|c|c|c|c|c|c|}
\hline COT & Defects & DR & IT & MOPT & UPDT & Response \\
\hline 0.003 & 0.05 & 10 & 0.007 & 7 & 0.03 & 54.74315 \\
\hline 0.005 & 0.05 & 20 & 0.005 & 6 & 0.016 & 33.72218 \\
\hline 0.005 & 0.1 & 10 & 0.007 & 7 & 0.016 & 53.10217 \\
\hline 0.005 & 0.1 & 10 & 0.007 & 7 & 0.03 & 53.35221 \\
\hline 0.005 & 0.05 & 10 & 0.005 & 6 & 0.03 & 54.39086 \\
\hline 0.005 & 0.05 & 20 & 0.007 & 7 & 0.016 & 33.30398 \\
\hline 0.003 & 0.1 & 20 & 0.005 & 6 & 0.03 & 29.28129 \\
\hline 0.005 & 0.05 & 10 & 0.005 & 7 & 0.016 & 53.78052 \\
\hline 0.005 & 0.1 & 20 & 0.007 & 6 & 0.016 & 31.36727 \\
\hline 0.003 & 0.05 & 20 & 0.005 & 6 & 0.016 & 32.35737 \\
\hline 0.005 & 0.1 & 10 & 0.005 & 6 & 0.016 & 53.43671 \\
\hline 0.003 & 0.1 & 10 & 0.005 & 6 & 0.03 & 54.41546 \\
\hline 0.005 & 0.1 & 20 & 0.007 & 7 & 0.03 & 29.98033 \\
\hline 0.003 & 0.1 & 10 & 0.007 & 6 & 0.03 & 54.41546 \\
\hline 0.003 & 0.05 & 20 & 0.007 & 6 & 0.03 & 31.08528 \\
\hline 0.005 & 0.05 & 10 & 0.007 & 7 & 0.016 & 53.78052 \\
\hline 0.005 & 0.1 & 20 & 0.007 & 6 & 0.03 & 30.18623 \\
\hline 0.005 & 0.1 & 20 & 0.005 & 6 & 0.03 & 30.18623 \\
\hline 0.005 & 0.05 & 20 & 0.007 & 6 & 0.016 & 33.72218 \\
\hline 0.003 & 0.05 & 10 & 0.007 & 6 & 0.016 & 54.84606 \\
\hline 0.003 & 0.1 & 20 & 0.005 & 6 & 0.016 & 30.4111 \\
\hline 0.005 & 0.1 & 20 & 0.005 & 6 & 0.016 & 31.36727 \\
\hline 0.003 & 0.05 & 20 & 0.007 & 7 & 0.016 & 32.0476 \\
\hline 0.003 & 0.05 & 10 & 0.007 & 6 & 0.03 & 55.12858 \\
\hline 0.005 & 0.1 & 20 & 0.005 & 7 & 0.016 & 31.14493 \\
\hline 0.003 & 0.1 & 20 & 0.007 & 6 & 0.016 & 30.4111 \\
\hline 0.003 & 0.05 & 20 & 0.005 & 7 & 0.03 & 30.8047 \\
\hline 0.005 & 0.1 & 10 & 0.005 & 6 & 0.03 & 53.70631 \\
\hline 0.003 & 0.1 & 10 & 0.005 & 6 & 0.016 & 54.13935 \\
\hline 0.003 & 0.05 & 20 & 0.007 & 6 & 0.016 & 32.35737 \\
\hline 0.005 & 0.05 & 20 & 0.007 & 6 & 0.03 & 32.33182 \\
\hline 0.003 & 0.1 & 20 & 0.005 & 7 & 0.03 & 29.1067 \\
\hline 0.005 & 0.05 & 10 & 0.007 & 7 & 0.03 & 54.03677 \\
\hline 0.003 & 0.1 & 20 & 0.007 & 6 & 0.03 & 29.28129 \\
\hline 0.005 & 0.05 & 10 & 0.005 & 7 & 0.03 & 54.03677 \\
\hline 0.003 & 0.1 & 20 & 0.005 & 7 & 0.016 & 30.22431 \\
\hline 0.005 & 0.1 & 10 & 0.007 & 6 & 0.03 & 53.70631 \\
\hline 0.005 & 0.05 & 10 & 0.007 & 6 & 0.016 & 54.11505 \\
\hline 0.005 & 0.05 & 10 & 0.007 & 6 & 0.03 & 54.39086 \\
\hline 0.003 & 0.05 & 10 & 0.005 & 6 & 0.03 & 55.12858 \\
\hline 0.003 & 0.05 & 20 & 0.007 & 7 & 0.03 & 30.8047 \\
\hline 0.003 & 0.1 & 20 & 0.007 & 7 & 0.016 & 30.22431 \\
\hline 0.005 & 0.05 & 20 & 0.005 & 7 & 0.03 & 31.96293 \\
\hline 0.005 & 0.1 & 10 & 0.007 & 6 & 0.016 & 53.43671 \\
\hline 0.003 & 0.1 & 10 & 0.007 & 7 & 0.03 & 54.03003 \\
\hline 0.003 & 0.05 & 20 & 0.005 & 6 & 0.03 & 31.08528 \\
\hline 0.003 & 0.05 & 10 & 0.005 & 7 & 0.016 & 54.48055 \\
\hline
\end{tabular}




\begin{tabular}{lcccccc}
\hline COT & Defects & DR & IT & MOPT & UPDT & Response \\
\hline 0.005 & 0.05 & 20 & 0.005 & 6 & 0.03 & 32.33182 \\
0.003 & 0.05 & 10 & 0.007 & 7 & 0.016 & 54.48055 \\
0.003 & 0.1 & 10 & 0.007 & 7 & 0.016 & 53.77384 \\
0.003 & 0.1 & 10 & 0.005 & 7 & 0.016 & 53.77384 \\
0.003 & 0.1 & 10 & 0.007 & 6 & 0.016 & 54.13935 \\
0.005 & 0.1 & 10 & 0.005 & 7 & 0.016 & 53.10217 \\
0.003 & 0.1 & 20 & 0.007 & 7 & 0.03 & 29.1067 \\
0.003 & 0.05 & 10 & 0.005 & 7 & 0.03 & 54.74315 \\
0.003 & 0.05 & 10 & 0.005 & 6 & 0.016 & 54.84606 \\
0.005 & 0.05 & 10 & 0.005 & 6 & 0.016 & 54.11505 \\
0.005 & 0.1 & 20 & 0.007 & 7 & 0.016 & 31.14493 \\
0.005 & 0.1 & 10 & 0.005 & 7 & 0.03 & 53.35221 \\
0.003 & 0.1 & 10 & 0.005 & 7 & 0.03 & 54.03003 \\
0.005 & 0.1 & 20 & 0.005 & 7 & 0.03 & 29.98033 \\
0.005 & 0.05 & 20 & 0.007 & 7 & 0.03 & 31.96293 \\
0.003 & 0.05 & 20 & 0.005 & 7 & 0.016 & 32.0476 \\
0.005 & 0.05 & 20 & 0.005 & 7 & 0.016 & 33.30398 \\
\hline
\end{tabular}

\section{Corresponding author}

Ahmed Deif can be contacted at: adeif@calpoly.edu 